\title{
Using object oriented technique to extract jujube based on landsat8 OLI image in Jialuhe Basin
}

\author{
Guotao Dong ${ }^{1, \text { a }}$, Mingyong Cai ${ }^{2, b}$, Dong Fan ${ }^{1,3, c}$, Wenwang Gao ${ }^{4,}$, Yaokang Lian ${ }^{5, e}$, Huijuan \\ Yin $^{1, \mathrm{f}}$, Suzhen Dang ${ }^{1, \mathrm{~g}}$ and Xinwei Guo ${ }^{1, \mathrm{~h}}$
}

${ }^{1}$ Yellow River Institute of Hydraulic Research, YRCC, MWR Key Laboratory of Soil and Water Loss

Process and Control in the Loess Plateau, Shunhe Road 45, Zhengzhou, China

${ }^{2}$ Satellite Environment Center of MEP, Fengde Road, Beijing, China

${ }^{3}$ Henan Polytechnic University, Shiji Road 2001, Jiaozuo, China

${ }^{4}$ Xifeng Soil and Water Conservation Experiment Station, South Street 268, Qingyang, China

${ }^{5}$ Research Center for Heihe River water resources and Ecological Protection, Qingyang Road 458, Lanzhou, China

adongguotao@hky.yrcc.gov.cn, bcaimingyong@126.com, ${ }^{c} 2210225173 @$ qq.com,

d888gww1107@163.com, ${ }^{\mathrm{d}} 290062957 @ q q . c o m$, fyinhuijuan@hky.yrcc.gov.cn,

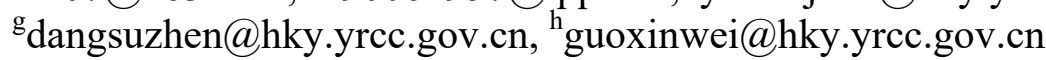

Keywords: Jujube, Landsat 8 OLI, object oriented technique, Jialuhe Basin.

\begin{abstract}
Vegetation is a crucial factor that affects watershed hydrological processes. To understand the effect of jujube vegetation variation on streamflow in the Jialuhe River Basin, jujube forest was investigated in this study. Object-oriented classification technique was used to extract jujube characteristics. The primary vegetation types were selected to sample the reflectance, and the spectral response curves were obtained. Then, the rule set of decision tree is constructed based on the comprehensive analysis of the image information and the object type. The classification results show that jujube forest is scattered and mainly distributed in the lower reaches. The area of jujube forest is $8.8 \mathrm{~km}^{2}$, which accounts for about $7.8 \%$ of the total area.
\end{abstract}

\section{Introduction}

Vegetation refers to forests, shrubs, grasslands, farmlands, and orchards, all of which are important components of the ecological cycle and help to maintain the environment. It is a crucial factor that affects watershed hydrological processes. Meanwhile, it is a key indicator of changes in the status of the environment, and so is also important globally to the biogeochemical balance of ecosystems [1].

It is a top priority for the way to extract vegetation changing data. Especially, the extraction of sub-category information of forest vegetation has always been a difficult point in remote sensing image classification. And it is more difficult to extract sub-category information of forest vegetation type only by taking advantage of the spectral information. Object-oriented classification was introduced in the 1970s. Since the mid-1990s, with an increase in availability of high spatial resolution images, the demand for object-oriented analysis has also increased [2]. As a widely-used 
method, object-oriented classification method is applicable to medium resolution remote sensing images at present.

Jialuhe River Basin is located in the middle reaches of Yellow River. Its primary problems have been soil and water erosion in addition to flood and sediment disasters. Few of the studies on jujube variation in the Jialuhe River Basin in the past have been focused. To understand the effect of the jujube vegetation variation on streamflow in the Jialuhe River Basin, jujube forest was investigated for this study. The primary objective is based remote sensing data to investigate and extract jujube characteristics in the Jialuhe River Basin.

\section{Study area}

Jialuhe River Basin is located in the Loess Plateau of China, between $109^{\circ} 59^{\prime} 10^{\prime \prime}-110^{\circ} 30^{\prime} 00^{\prime \prime} \mathrm{E}$ and $37^{\circ} 59^{\prime} 18^{\prime \prime}-38^{\circ} 28^{\prime} 25^{\prime \prime} \mathrm{N}$, and is a tributary of the Yellow River, with a drainage area of $1134 \mathrm{~km}^{2}$. The main channel length is $93 \mathrm{~km}$. The climate is semi-arid, with an average annual precipitation of $407 \mathrm{~mm}$, of which $74 \%$ falls in the period of June to September. Topography in this basin is characterized by upland, gently sloping ridges, steep hillslopes and well-defined alluvial valleys with incised channels that end at an actively eroding head cut (Fig.1). The primary soil types are loess mantle, and silty clay loam textures. Land-use types are mostly grassland, farmland, and sandy land.

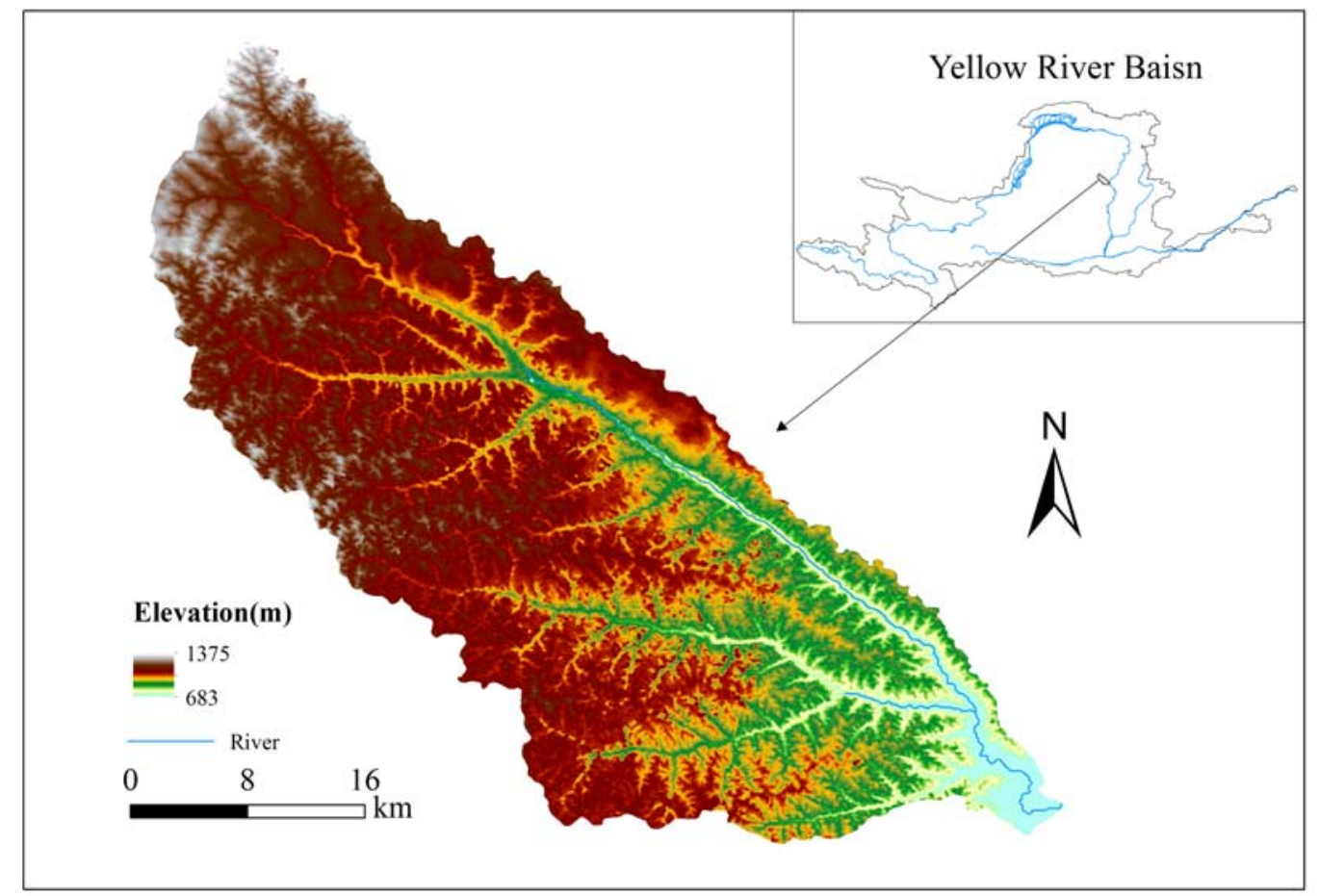

Fig.1 Location and DEM of study area

\section{Methods}

\subsection{Data sources}

NASA's Landsat Program-LandSat8 OLI was used to ground feature identification and 
information extraction [3]. The LandSat8 OLI data covering the entire study area were acquired on Jul. 1, 2015. The images were taken when vegetation was growing well and could fully represent the growing conditions of the vegetation.

\subsection{Data processing}

Remote sensing data were processed and analyzed using ENVI 4.8 image processing software. The original TM/ETM images were georeferenced to the Albers projection system using the nearest-neighbor resampling method. To normalize the coordinate system, a geometric correction was completed for the TM/ETM image on the basis of a 1:50,000 digitized map.

\subsection{Object-oriented classification}

Object-oriented image classification has tremendous potential to improve classification accuracies of land use and land cover (LULC). The combination of segmentation into image objects substantially improved classification accuracy for the scene compared to a traditional pixel-based method. Object-oriented classification does not operate directly on single pixel, but objects consisting of many pixels that have been grouped together in a certain way by image segmentation.

Image segmentation is a preliminary step in object-oriented image classification that divides the image into homogeneous, contiguous objects. Image segmentation techniques can be grouped into three types: thresholding/clustering, edge based, and region based[4]. Image segmentation techniques make use of both spectral information (feature vector of the pixels) and spatial information (size, shape and adjacency to other pixels) to identify and delineate suitable segments within an image.

Edge-based segmentations attempt to find segment boundaries by detecting edges between image areas with different characteristics. Subsequently, image regions that are completely surrounded by edge pixels become segments. As a result, the image pixels can either belong to a segment or form a boundary. The main disadvantage of this method is related to the fact that small terrain objects are completely obscured by boundary pixels. In the present study an edge-based segmentation was adopted. More details can be found in Fu and Mui [4], and de Bruin and Molenaar [5].

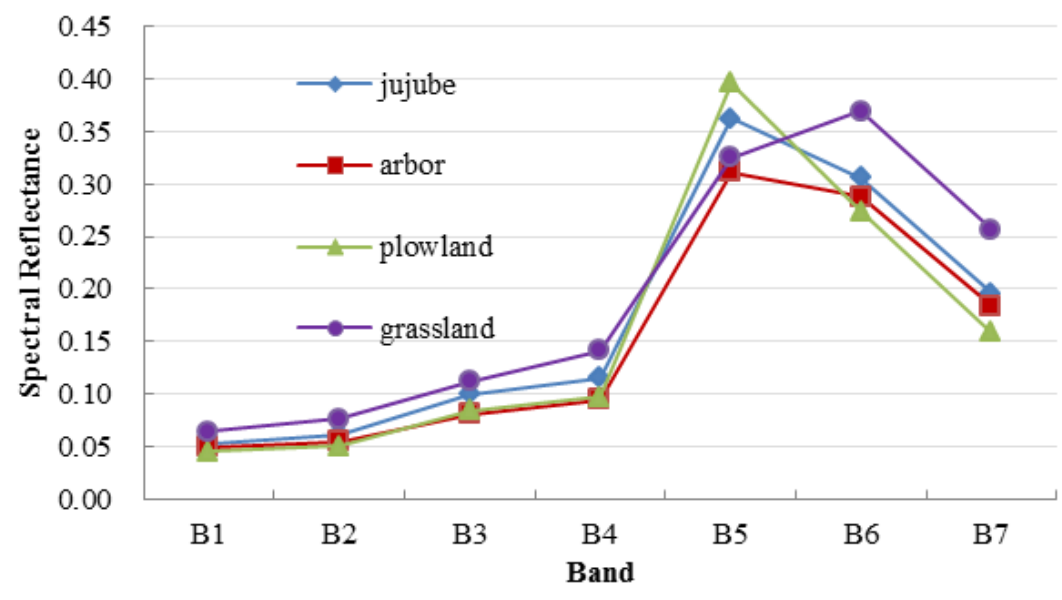

Fig. 2 Spectral curves of different land cover types

\subsection{Spectral curves and decision tree of classification}

According to the classification rules, the primary vegetation types were selected to sample the 
reflectance, and the spectral response curves were obtained (Fig.2). On the basis of the spectral curve and spatial characteristics, the rule set of decision tree is constructed based on the comprehensive analysis of the image information and the object type (Fig.3).

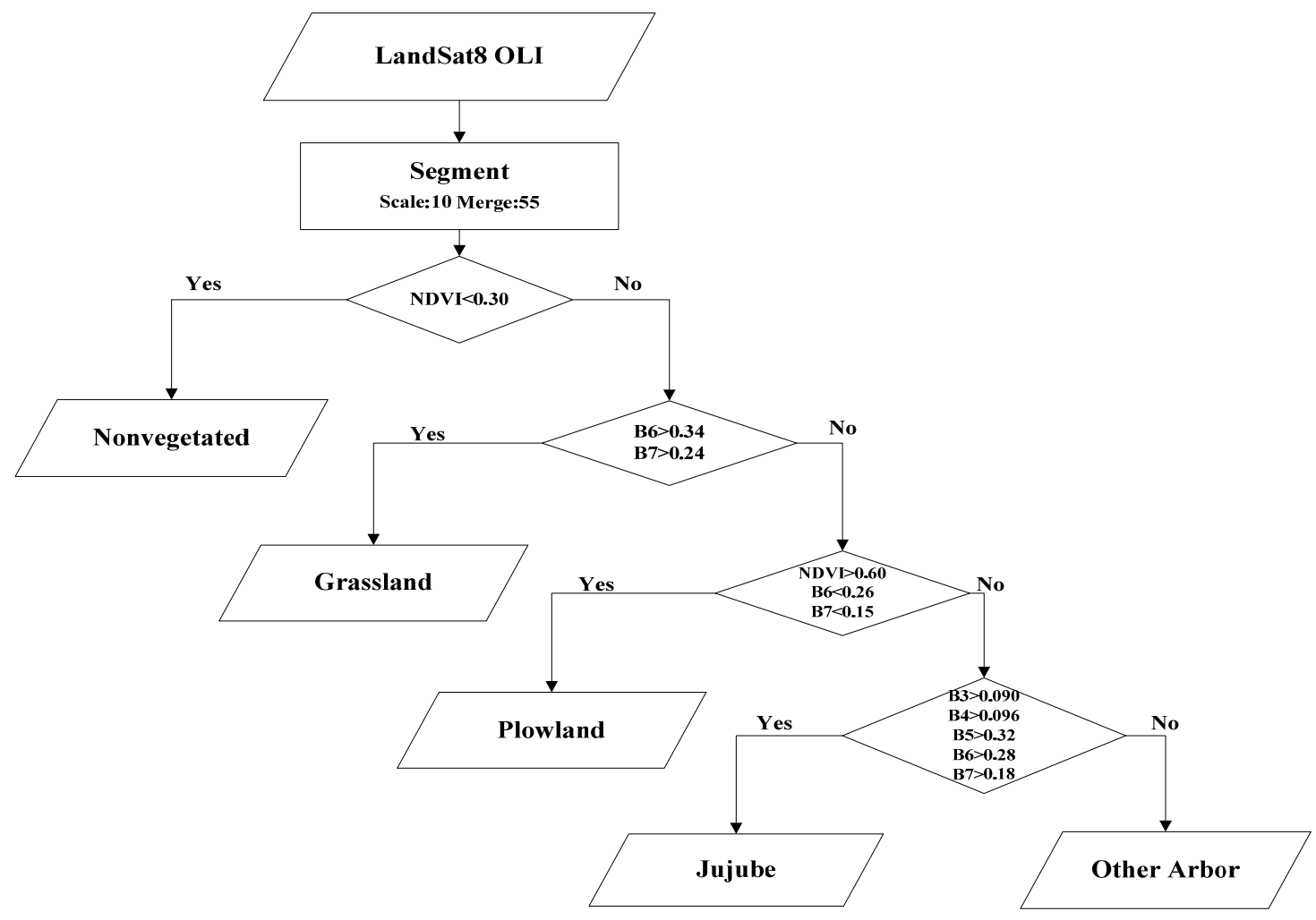

Fig 3 Decision tree of classification

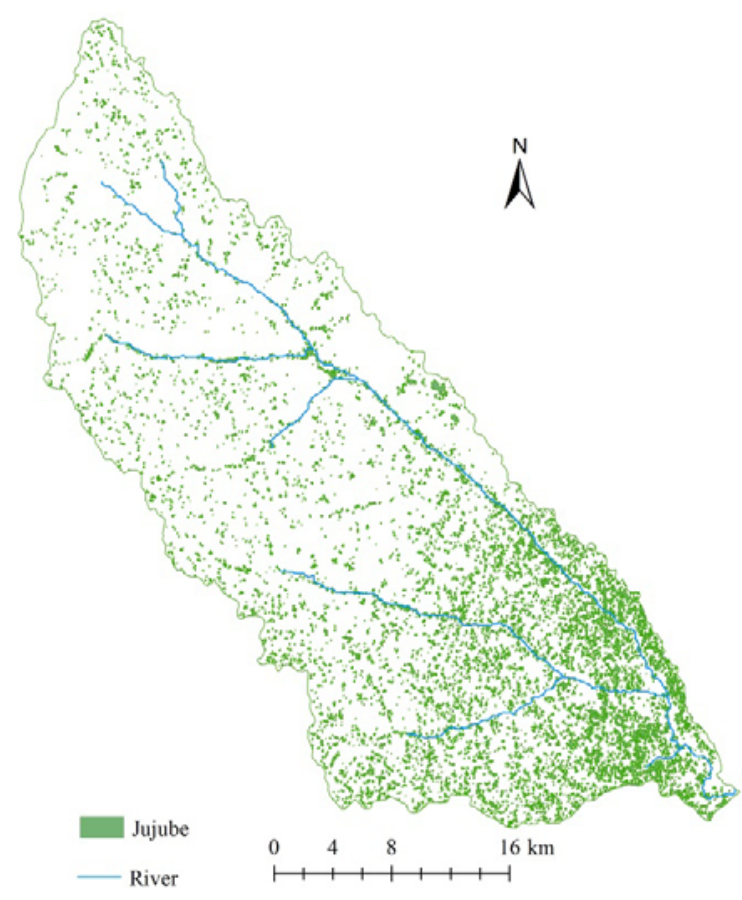

Fig. 4 The extraction results of Jujube in Jialuhe River Basin 


\section{Results}

The jujube forest maps produced from Landsat OLI images were displayed in Fig.4. The classification results (Fig. 4) show that jujube forest was scattered and mainly distributed in the lower reaches. The area of jujube forest is $8.8 \mathrm{~km}^{2}$, accounting for about $7.8 \%$ of the total area, which is similar to the statistical data.

\section{Summary}

The classification results show that jujube forest was scattered and mainly distributed in the lower reaches. The area of jujube forest is similar to the statistical data. It indicated object-oriented classification technique was suitable to extract jujube characteristics.

\section{Acknowledgements}

This work was financially supported by the National Natural Science Foundation of China (Grant No. 41301496, 41301030), National Key Research and Development Program (2016YFC0402409), and National Key Technology R\&D Program (2012BAD15B05).

\section{References}

[1] Niemi G.J. and McDonald M.E., Application of Ecological Indicators. Annual Review of Ecology, Evolution, and Systematics, vol 35, 89-111(2004).

[2] De Kok R, Schneider T, Ammer U, Object based classification and applications in the alpine forest environment. Int Arch Photogramm Remote Sens vol 32, Part 7-4-3 Wg, Valladolid, Spain, 3-4. June (1999).

[3] Roy D. P, Wulder M A, Loveland T R, et al., Landsat-8: Science and product vision for terrestrial global change research. Remote Sensing of Environment, 145:154-172 (2014).

[4] Fu, K. S., and Mui, J. K. A survey on image segmentation. Pattern Recognition, 13:3-16 (1981).

[5] De Bruin, S and Molenaar, M, Remote sensing and geographical information systems.In Spatial Statistics for Remote Sensing, edited by A. Stein, F. van der Meer and B. Gorte (Dordrecht: Kluwer Academic), pp. 41-54 (1999). 